\title{
Hydrophobic and raw glass beads flow behavior under different processing conditions
}

\author{
Shirin Enferad ${ }^{1,2, *}$, Salvatore Pillitteri ${ }^{3}$, Geoffroy Lumay $^{3}$, Claire Gaiani ${ }^{2}$, Sebastien Kiesgen De Richter ${ }^{1}$, Michaël \\ Marck $^{3}$, Syrym Umbetov ${ }^{1}$, Nicolas Vandewalle ${ }^{3}$, and Mathieu Jenny ${ }^{1}$ \\ ${ }^{1}$ Université de Lorraine, Laboratoire d'Energétique et de Mécanique Théorique et Appliquée (LEMTA), CNRS, UMR 7563, \\ Vandoeuvre-lés-Nancy, F-54500, France. \\ ${ }^{2}$ Université de Lorraine, Laboratoire d'Ingénierie des Biomolécules (LIBio), 2 avenue de la Forêt de Haye, TSA 40602,54518 \\ Vandoeuvre-lès-Nancy, France. \\ ${ }^{3}$ GRASP, Institute of Physics B5a, University of Liège, B4000 Liège, Belgium.
}

\begin{abstract}
Controlling the powder flow by the surface properties of the particles as well as understanding their flowability under different processing dynamics are amongst the major challenges of the powder industry. Indeed, handling large quantities needs powders with good flowability, adequate compressibility and few electrostatic charges. We have performed a chemical treatment in order to obtain hydrophobic glass bead and its bulk behavior has been compared with raw glass bead. We characterized flow properties under different processing conditions. Both powders presented similar flow behavior in the free fall measurement, however hydrophobic glass bead showed lower flow behavior under flow with rotating drum. Also, hydrophobic glass bead presented negative and raw glass bead almost zero electrostatic charges.
\end{abstract}

\section{Introduction}

Powders are omnipresent in our daily life and they are extensively used in various industries such as pharmaceutical, cosmetics, chemical, food and construction. Despite numerous studies on powder rheology [1-3], the behavior of powders is not fully understood, due to their complexity. In fact, the flow behavior of a powder depends on many factors including surface properties, size, shape of particles [4], etc.

However, understanding the powder's flowability is one the most important objectives in powder industries. Indeed, it can allow them to anticipate the powder's characteristics by knowing the conditions that they encounter during process. In order to identify the pertinent parameters governing the powder's flow, using different methods are always very beneficial approach. They can provide different point of view and indices to assess powder flowability with several mechanical and dynamical conditions explained in the following.

The free fall test is one of the inexpensive, efficient and rapid method to measure powder behavior without imposing any external force. The measurement is just based on the powder free fall due to the gravity. The flowability of powder is measured only by averaging time for which the constant volume of the powder pass through the measuring equipment [5].

Apparition of powder electrostatic charges as a result of particle-particle interaction and powders interaction with manipulating equipment during powder process

*e-mail: shirin.enferad@univ-lorraine.fr\&shirinenferad@yahoo.com

A video is available at https://doi.org/10.48448/ryyr-1y61 is a know phenomena in industries. An increase in powder electrostatic charge can lower its flowability [6]. Whereby, Powder characterization based on their electrostatic charge is of great interest [7].

In addition, rotating drum is one of the efficient equipments to characterize macroscopic properties of powders under aeration condition. In this equipment, drum rotates around its central axis with different preselected speed and fluidize the powder inside drum. Then, it reports powder cohesion and repose angle based on fluctuation of powderair interface inside drum as a result of different rotation speed [3].

This paper focuses on studying the influence of formulation on powder flowability and evolution of powder flowability under different processing conditions. Therefore, first a hydrophobic glass bead has been prepared then its flow behavior has been compared with raw glass bead. The Granutools equiement have been used for analyzing powder behavior and finally the results have been compared.

\section{Materials and methods}

In this section, the materials and implemented three different methods for characterizing powders have been explained. The purpose of using different methods was to characterize the powders under different processing conditions and to observe the influence of formulation on powders flowability. Therefore, three series of characterization measurements have been performed on raw and hydrophobic glass beads by implementing Granutools equipment. 
The powders flowability has been measured with Granuflow and GranuDrum equipment and their electrostatics charge has been studied with GranuCharge.

\subsection{Powders preparation and conditioning}

In this study glass bead of types S 90-150 $\mu \mathrm{m}$ has been used. Hydrophobic surface treatment has been done on raw glass bead. With the purpose of preparing hydrophobic surface formulation on glass bead, first the hydrophilic surface treatment has been performed [8] then it has been followed with silanization. The silanization of glass beads with mixture of toluene $(500 \mathrm{~mL})$ and $1 \mathrm{H}, 1 \mathrm{H}, 2 \mathrm{H}, 2 \mathrm{H}-$ perfluorooctyltriethoxysilane $(2.5 \mathrm{~g})$ has been performed, this mixture has been kept maximum $72 \mathrm{~h}$ under extractor hood at ambient temperature and then the powder filtered and washed with pure toluene and finally they kept about $24 \mathrm{~h}$ under extractor hood for evaporating the toluene inside the powder and finally with the purpose of completely drying the powder, it has been kept in an oven, maximum $4 \mathrm{~h}$ at $70{ }^{\circ} \mathrm{C}$.

\subsection{GranuFlow}

Figure 1 shows GranuFlow equipement that measures the flowability of a granular material. The powder to analyze is poured in a steel tube to avoid electrostatic charges accumulation. The opening of the bottom is obstructed with a hole-pierced disk. Each hole of the disc has a different diameter aperture $d$. Below, a scale is placed and measures the flow rate of the powder through the opening. By measuring the different mass flow rates $F$ depending of the size aperture $d$, one can obtain the flow index $C$ using the Beverloo model:

$$
F=C \sqrt{g}\left(d-d_{\min }\right)^{2.5} .
$$

The flow index $C$, expressed in $\mathrm{g} / \mathrm{cm}^{3}$ can be interpreted as the ability of a powder to flow and $g$ is the gravity of earth $\left(9.81 \mathrm{~m} / \mathrm{s}^{2}\right)$.

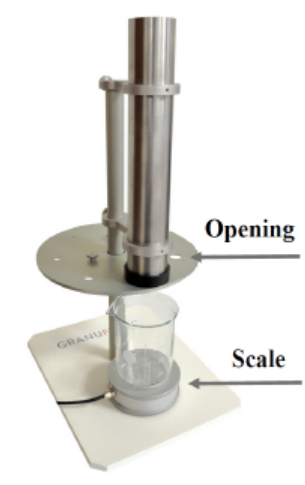

Figure 1. Diagram of GranuFlow@ equipment. It is equipped to with cylindrical stainless steel tube and a disk with 7 different diameter size openings through which the powder pass from each opening one by one and it fall into a container. A scale placed under container measures continuously the powder mass.

\subsection{GranuCharge}

Electrostatic charges are created due to the triboelectric effect inside a powder during a flow; meaning that the particles exchange charge at the result of contact with the other particles and devices wall. GranuCharge equipment is illustrated in figure 2 , it is a very useful equipment to measure the ability of a flowing powder to be charged electrostatically. It measures electrostatic charge of the powders by putting them on motion under the influence of gravity. In this measurement, the experimental set up is consist of three parts: an stainless-steel pipe, V-tube and a Faraday cup. The stainless-steel pipe is for feeding the V-tube by automatic rotation inside the tube.

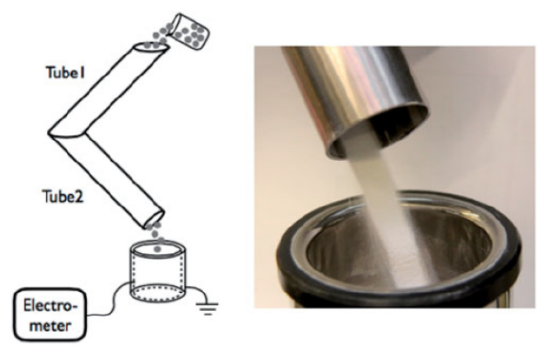

Figure 2. Left: Sketch of GranuCharge used to measure the electrostatic charge created inside a powder after flow in contact with a selected material. Right: Picture of the powder flowing from the tube 2 into the Faraday cup measuring the electrostatic charge [6].

After feeding the tube by the stainless-steel pipe the powder flow inside V-tube and at the end it fall into the faraday cup which is connected to an electrometer to measure the powder charge obtained during flow; before starting each test the whole assembly has been connected to the earth to be sure that they are discharged. The electrometer is capable of measuring charge ranging $0.1 \mathrm{nC}-1 \mu \mathrm{C}$. The powder charge density is computed by dividing calculated charge by the mass of powders. The charge density unit is Coulomb per kilogram.

\subsection{GranuDrum}

The rotating drum is one of the most extensively used practical geometry to study the flow behavior of granular material [3]; especially non cohesive powders. The experimental set up consists of a drum with two glass walls which rotates around its central axis with the angular velocity $\Omega$. The flowing dynamics of powders inside the drum is function of this rotating angular velocity. The drum is generally half filled with the powder and back-lighted while a CCD camera takes pictures of the powder-air interface position inside the drum. For each imposed angular velocity, 50 pictures are taken, separated by $0.5 \mathrm{~s}$. The average powder-air interface position is computed by image processing analysis. From this analysis, two measures are extracted: the cohesion index $\sigma_{f}$, which is the fluctuations of the powder-air interface and is representative of the cohesion of granule inside the drum, and the flowing angle 
$\alpha_{f}$, which is the angle between the horizontal and the average interface. In this study, the rotating angular velocity has been selected between 2-30 rpm.

\subsection{Environmental control}

Powder aging happens as a result of influence of some factors such as temperature and humidity. Where powder characteristics can change by aging. As an instance, high air humidity can increase inter-particle cohesion consequently it lowers the powder flowability. It has been reported that the best experimental condition for glass bead corresponds up to $45 \%$ of air humidity where cohesion is minimum [9], however if the air humidity increases more than $70 \%$ then an strong aging effect is expected [10]. In addition, temperature is another factor in powder aging that can influence the flow behavior of powders in different ways. By increasing the temperature, the water content at the surface of the particle decrease which lowers the surface cohesion by lowering the liquid bridge between particles. Also an increase in temperature can exceed the glass transition temperature of material therefore it modifies the texture of the latter [11]. In our study in order to avoid aging effect, the humidity and temperature have been kept constant at $37-44 \%$ and $22{ }^{\circ} \mathrm{C}$, respectively, where the cohesion is minimum and the powders are almost in dry situation.

\section{Results and discussion}

\subsection{Flowability}

The flowability of raw and hydrophobic glass beads have been studied with the GranuFlow. Each powder flow rate has been measured for several aperture, from $2 \mathrm{~mm}$ to 16 $\mathrm{mm}$ in standard conditions of temperature and humidity. The results are shown on Figure 3. As one can see, the flow properties of hydrophobic and raw glass beads are similar. The flowing index obtained from the fit of Eq. (1) are respectively $C=8.62 \pm 0.04 \mathrm{~g} / \mathrm{cm}^{3}$ and $C=8.89 \pm 0.04$ $\mathrm{g} / \mathrm{cm}^{3}$ for raw and hydrophobic glass beads.

\subsection{Charge}

Figure 4 represents the results corresponding to electrostatic charge measurement of raw and hydrophobic glass beads. Three values have been collected for each samples, these values are consisting of initial charge $\mathrm{q}_{0}$, final charge $\mathrm{q}_{f}$ and $\Delta \mathrm{q}=\mathrm{q}_{f}-\mathrm{q}_{0}$. The obtained results show that both powders had almost zero electrical charge as initial charge, after flow inside stainless steel V-tube, raw glass bead kept this tendency to having almost zero charge however hydrophobic glass bead obtain negative charge. The quantity of each sample was $50 \mathrm{~mL}$ during measurement. In general, a powder can obtain negative or positive charge after flow, based on their tendency to obtain or loss electron [6]. As an instance, in a study on lactose powder it has been observed that at low relative humidity lactose powder charges positively while at higher relative humidity it charged negatively in fact the relative humidity here

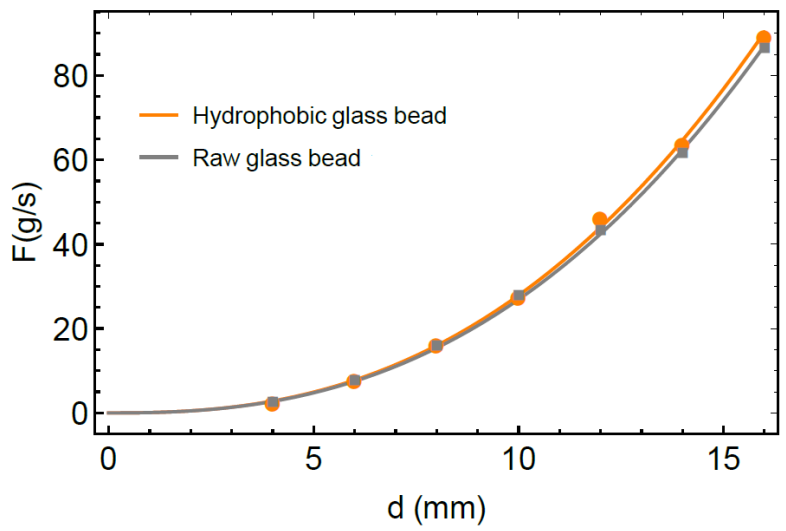

Figure 3. Fit of the Eq. (1) on the data for respectively the raw bead, in grey, and hydrophobic bead, in orange. The humidity and temperature range are $37-44 \%$ and $22{ }^{\circ} \mathrm{C}$, respectively.

played role in tendency of powder to be charged with different sign [12]. The powder charge can vary by varying the material that they contact during process. In addition, it has been reported that the surface formulation of powders or addition of additive can change the sign of particle charge or modify the quantity of charge [12]. In the same study on lactose powder they noticed that by adding syloid 244FP to the lactose powder it gets negative final charge, while by addition syloid XDP 3050 to lactose powder it gets positive charge also by increasing the quantity of both additives the charge of both powders have started to decrease. Here in our study, raw glass bead presented zero electrostatic charge while after hydrophobic formulation it obtained negative charge.

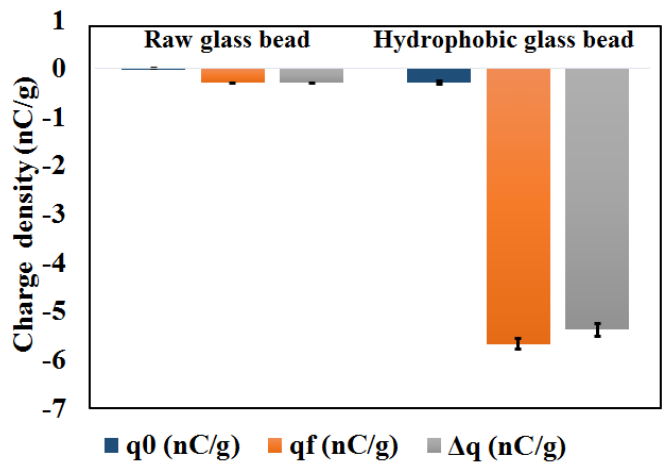

Figure 4. The evolution of electrostatic charge of raw and hydrophobic glass beads before and after flowing inside V-tube. The measurement performed by GranuCharge equipment. The humidity and temperature range are $37-44 \%$ and $22{ }^{\circ} \mathrm{C}$, respectively.

\subsection{Cohesion}

Figure 5 shows evolution of the cohesion $\sigma_{f}$ and the flowing angle $\alpha_{f}$ of raw and hydrophobic glass beads. It has 
been observed that cohesion of both samples have been enhanced by increasing rotating speed of the drum, meaning that their flowability decreases under motion, particularly after $10 \mathrm{rpm}$. Hydrophobic glass bead showed slightly more cohesion than raw glass bead. This could be explained with the GranuCharge measurements. Hydrophobic glass bead presented more electrostatic charge than raw glass bead. This can lead to higher cohesion of hydrophobic glass bead under motion (see figure 4).

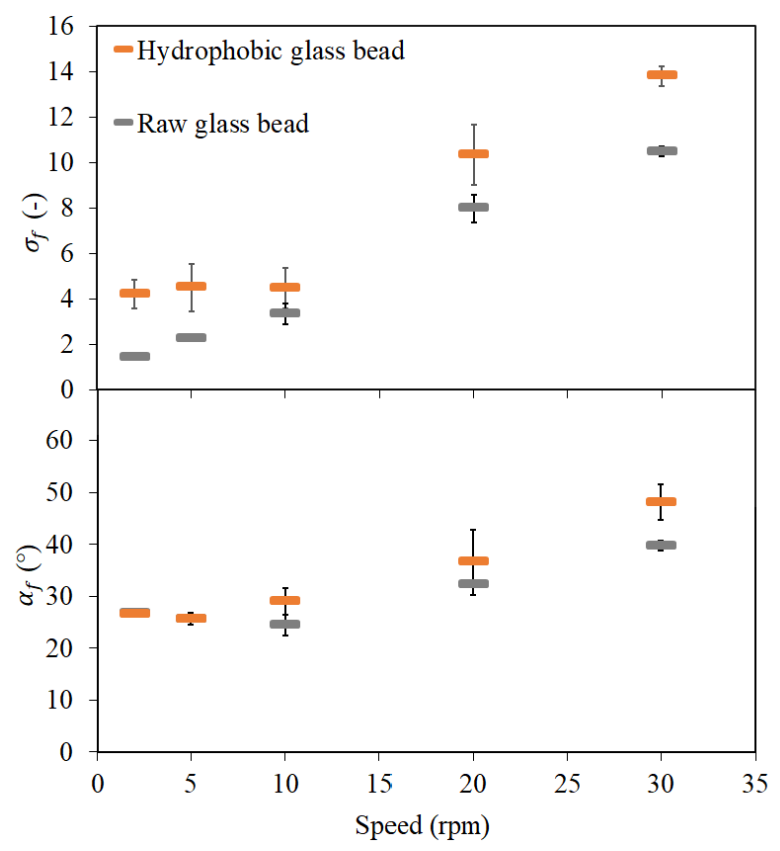

Figure 5. Evolution of the flowing angle and cohesion of raw and hydrophobic glass beads under different rotational speed with the measurements performed by GranuDrum. The humidity and temperature range are $37-44 \%$ and $22{ }^{\circ} \mathrm{C}$, respectively.

\section{Conclusion}

This paper presented a study on flow behavior of two types of powders consisting of raw glass bead and hydrophobic surface formulated glass bead. The objective was to explore the behavior of hydrophobic formulated glass bead under different processing dynamics and compare it with the same measurements done on raw glass bead. Therefore, the behavior of these two powders have been studied under different processing conditions consisting of free fall test, rotating drum test and electrostatic charge measurement. Based on GranuFlow measurements, it has been observed that the powders have similar flowability. However, the GranuCharge measurement represented a charge difference between hydrophobic and raw glass bead, it re- ported negative charge for hydrophobic glass bead and almost zero charge for raw glass bead which was expected to has influence on their flow behavior. This issue has been confirmed with the cohesion increase in hydrophobic glass bead under motion by measurement performed with GranuDrum equipment. GranuDrum reported an increase in cohesion of both raw and hydrophobic glass bead under motion, while hydrophobic glass bead showed more increase in cohesion than raw glass bead, meaning that the flowability of these two powders decreases under motion with more decrease in hydrophobic glass bead. we guess this can be due to an internal effect such as apparition of electrostatic charge by increasing rotation speed of drum.

Acknowledgments - This study is conducted in the framework of the "PowderReg" project, funded by the European program Interreg VA GR within the priority axis 4 "Strengthen the competitiveness and the attractiveness of the Grande Région / Großregion".

\section{References}

[1] E. Guerin, P. Tchoreloff, B. Leclerc, D. Tanguy, M. Deleuil, G. Couarraze, Int. J. Pharm 189(1), 91-103 (1999)

[2] C. Salameh, J. Scher, J. Petit, C. Gaiani, C. Hosri, S. Banon, J. Powder Technol 292, 307-313 (2016)

[3] G. Lumay, F. Boschini, K. Traina, S. Bontempi, J.-C. Remy, R. Cloots and N. Vandewalle, J. Powder Technol 224, 19-27 (2012)

[4] A. Castellanos, Adv. Phys 54(4), 263-376 (2005)

[5] A. Hassanpour, C. Hare, M. Pasha, Powder flow: Theory, characterisation and application (Royal Society of Chemistry 2019)

[6] A. Rescaglio, J. Schockmel, N. Vandewalle and G. Lumay, Combined effect of moisture and electrostatic charges on powder flow, in EPJ Web of Conferences. EDP Sciences 140, 13009 (2017)

[7] DA. Hays, J. Mason, PJ. Mason, RE. Zeman, D. Jackson, US Patent App.15/050,729 (2016).

[8] S. Enferad, J. Petit, C. Gaiani, V. Falk, J. Burgain, S. Kiesgen De Richter and M. Jenny, Part. Sci. Technol, 1-9 (2020)

[9] N. Vandewalle, G. Lumay, F. Ludewig, J. Fiscina, Phys. Rev. E 85(3), 031309 (2012)

[10] N. Fraysse, H. Thomé, L. Petit, Eur. Phys. J. B 11(4), 615-619 (1999)

[11] JJ. Fitzpatrick, M. Hodnett, M. Twomey, PSM. Cerqueira, J. O'flynn, YH. Roos, Powder Technol 178(2), 119-128 (2007)

[12] G. Lumay, S. Pillitteri, M. Marck, F. Monsuur, T. Pauly, Q. Ribeyre, F. Francqui and N. Vandewalle, J Drug Deliv Sci Technol 53, 101192 (2019) 